\title{
Cáncer de vesícula biliar: Tendencia y distribución del riesgo en Chile
}

\author{
Marcelo Andia $K^{1 a}$, Alessandra Gederlini $G^{\mathbf{1 b}}$, \\ Catterina Ferreccio $\mathbf{R}^{\mathbf{1}}$. \\ Gallbladder cancer: \\ trend and risk distribution in Chile
}

Background: Gallbladder cancer (GBC) is the first cause of death by cancer among Chilean women and mortality has not improved in the last 20 years. Aim: To study GBC mortality trend from 1985-2002, analyze risk differentials by age, sex, geographic region and accessibility to surgery. Material and Methods: Mortality data was obtained from death certificate databases. Population data was obtained from the census and biliary surgery information, from Ministry of Health registries. Standardized Mortality rates were based in the world population; trend was analyzed with point of change methods. Results: From 1985 to 2002, 27,183 GBC deaths occurred, 1,510 per year. The absolute number of deaths increased in $65 \%$ but standardized mortality rates remained unchanged at 11.3 per 100,000. These were higher among women than men (15.6 and 7.0, respectively, with a risk ratio of 2.2). Sex ratio peaked at ages 35-54 with risk ratio of 4.1. Death risk increased from North to South, peaking in poorer areas, especially in places with rural population and Mapuche ethnic admixture. Mortality appear to correlate with the rate of people waiting for gallbladder surgery, but not reaching statistical significance ( $\mathrm{r}^{2} 0.27$, ns). Compared with other countries, Chile has a higher rate of GBC deaths in relation to its gross domestic product per capita. Conclusions: There is a high and persistent persistent risk for GBC in Chile, particularly among women, from the Southern regions where gallbladder surgery is insufficient for the needs, as reflected by the rates of people waiting for biliary surgery. GBC rates could be dropped by offering gallbladder surgery to everyone waiting for it and to those incident cases with gallstones (Rev Méd Chile 2006; 134: 565-74).

(Key w ords: Cholecystectomy; Gallbladder neoplasms; Gallstones)

Recibido el 17 de agosto, 2005. Aceptado el 15 de noviembre, 2005.

${ }^{1}$ Departamento de Salud Pública, Facultad de Medicina, Pontificia Universidad Católica de Chile.

anterno, Facultad de Medicina, Pontificia Universidad Católica de Chile

bioestadística

Correspondencia a: Dra. Catterina Ferreccio R. Departamento de Salud Pública, Facultad de Medicina, Pontificia Universidad Católica de Chile. Marcoleta 434, Santiago, Chile. Fax: (2) 633 1840. E mail: cferrec@med.puc.cl 
$\mathrm{E}$ cáncer de vesícula biliar (cáncer vesicular) es la primera causa de muerte oncológica en las mujeres chilenas, seguido por el cáncer de mama, de estómago y cérvico uterino. Este mismo grupo presenta una de las tasas de mortalidad más altas a nivel mundial, por lo que esta patología es una prioridad sanitaria y científica en Chile.

El cáncer vesicular presenta muy baja incidencia en los países desarrollados y no se incluye en las principales bases de datos internacionales de cáncer, IARC ${ }^{1}$ y $\mathrm{OMS}^{2}$. Las poblaciones de bajo riesgo de cáncer vesicular están en el norte de Europa y entre la población blanca no-hispánica de Estados Unidos; las poblaciones de muy alto riesgo, están en los países andinos -particularmente Chile y Bolivia- y entre la población hispana e indígena de Norteamérica. Las poblaciones de bajo riesgo reportan tasas de incidencia alrededor de 1,3 casos por 100.000 habitantes (h) en mujeres y de 0,8 casos por $100.000 \mathrm{~h}$ en hombres ${ }^{3,4}$; las de alto riesgo, reportan tasas de incidencia de 10 a 15 casos por $100.000 \mathrm{~h}$ en mujeres y entre 3,5 y 7,0 casos por $100.000 \mathrm{~h}$ en hombres ${ }^{4}$. Chile sobrepasa estas últimas cifras, alcanzando tasas de incidencia de 28 casos por $100.000 \mathrm{~h}$ en mujeres y 9,4 casos por $100.000 \mathrm{~h}$ en hombres en la provincia de Valdivia en $2002^{5}$.

La mortalidad por cáncer vesicular entre los años 1999 y 2000, estandarizada por edad y sexo según la distribución de población mundial, fue entre 0,7 y 1,6 muertes por $100.000 \mathrm{~h}$ en Estados Unidos y la Comunidad Europea ${ }^{6,7}$; de 3,5 a 16,0 muertes por $100.000 \mathrm{~h}$ en los países de alto riesgo ${ }^{4}$; Chile se ubica en este último grupo presentando 7,0 muertes por $100.000 \mathrm{~h}$ en hombres y 15,6 muertes por $100.000 \mathrm{~h}$ en mujeres en 2002.

Las principales hipótesis explicatorias para este patrón geográfico han sido genéticas (mayor susceptibilidad en población amerindia), hormonales (relacionadas con el estrógeno) y ambientales (dieta, pobreza, infecciones) ${ }^{4,8-11}$. El objetivo del presente trabajo es actualizar la situación epidemiológica del cáncer vesicular en Chile, a través del análisis de la tendencia de la mortalidad por cáncer vesicular entre 1985 y 2002 y su distribución por edad, sexo y región. Un segundo manuscrito presentará un análisis geográfico de los factores de riesgo a nivel comunal.

\section{MATERIAL Y MÉTODO}

Fuentes de información. Los datos de mortalidad de 1985-2002 se obtuvieron de las bases de datos de defunciones del Ministerio de Salud ${ }^{12}$; se seleccionó las muertes con el código 156 de la CIE 9 «umor maligno de la vesícula y vías biliares extra hepáticas» (correspondiente a los códigos C23 y C24 de la clasificación CIE10). Los datos de población se obtuvieron del censo y de las proyecciones del Instituto Nacional de Estadísticas ${ }^{13}$. Las tasas de mortalidad por cáncer vesicular de otros países se obtuvieron de diversas publicaciones $^{3,4,6,7}$. El Producto Interno Bruto (PIB) per cápita se obtuvo del World Factbook ${ }^{14}$. La información sobre colecistectomías y pacientes en espera de cirugía biliar por Servicio de Salud, se obtuvo de la Secretaría del Plan Auge del Ministerio de Salud ${ }^{12}$.

Estadística. Las tasas de mortalidad se estandarizaron en base a la distribución de la población mundial propuesta por Doll et $\mathrm{al}^{15}$, y se presentan los intervalos de confianza de 95\% para dichas tasas. La tendencia temporal de la mortalidad en el período 1985-2002, se analizó con el método de regresión de punto de cambio (joinpoint regression) propuesto por Kim et al $^{16}$ usando el programa Joinpoint ${ }^{17}$. La asociación entre la mortalidad estandarizada por cáncer vesicular y el PIB per cápita y la asociación entre tasa de mortalidad y tasa de listas de espera se estudiaron mediante regresiones con mínimos cuadrados, usando el paquete estadístico SPSS $13^{18}$.

\section{RESULTADOS}

La tasa bruta de mortalidad por cáncer vesicular chilena aumentó de 9,4 muertes por $100.000 \mathrm{~h}$ en 1985 a 12,0 muertes por $100.000 \mathrm{~h}$ en $2002^{12}$. En los hombres, en el mismo periodo, el aumento fue de 4,7 a 6,2 muertes por $100.000 \mathrm{~h}$ y en las mujeres de 14,0 a 17,8 muertes por $100.000 \mathrm{~h}$. Aunque el número promedio de muertes en el período aumentó en $65 \%$, la tasa de mortalidad ajustada por edad y sexo se mantuvo estable en el período en torno a 11,3 muertes por $100.000 \mathrm{~h}$ (95\% intervalo de confianza, IC: 10,5-12,1); en los hombres, la tasa estandarizada promedio fue de 
7,0 muertes (95\% IC: 6,3-7,8) y en las mujeres de 15,6 (95\% IC: 14,4-16,7) (Tabla 1). El análisis de punto de cambio sugiere una tendencia a la disminución de las tasas de mortalidad de los hombres a partir de 1994 y de las mujeres a partir de 1990. Sin embargo, la tendencia de la tasa de mortalidad estandarizada se ajustó a un modelo de regresión Poisson sin puntos de cambio y las pendientes de las tendencias, tanto en hombres como en mujeres, no fueron significativas $(\Omega=0,0)$, indicando que la mortalidad permaneció estacionaria en el período estudiado (Figura 1).

La tasa de mortalidad específica en mujeres y hombres aumentó con la edad, alcanzando su máximo en los mayores de 75 años, con una tasa de muertes por $100.000 \mathrm{~h}$ de 107,8 en hombres (95\% IC: 103,5-112,1) y de 163,1 muertes en mujeres (95\% IC: 159,0-167,0) (Tabla 2 y Figura 2). En todas las edades, el riesgo de las mujeres fue mayor que el de los hombres, sin embargo, el exceso de riesgo de las mujeres varía con la edad, llegando al máximo $\mathrm{RR}=4,1$ (95\% IC: 1,2-14,1) entre los 35 y 54 años y luego desciende progresivamente hasta estabilizarse en $\mathrm{RR}=1,5$ (95\% IC: 1,2-1,9) en las mayores de 75 años (Figura 2). El exceso de riesgo en las mujeres se mantuvo estable en el periodo 1985-2002, con un RR promedio de 2,2 (95\% IC: 2,0-2,5).

La distribución geográfica del cáncer vesicular fue heterogénea, con seis regiones bajo el riesgo promedio nacional, las regiones I, II, III, V, RM y XII, con una tasa media de mortalidad de 9,9 por 100.000 h, (IC 95\%: 9,6-10,1). Las más bajas fueron las regiones RM, II, V y XII, que corresponden a las regiones con mayor nivel de ingreso del país ${ }^{19}$. Las regiones IV, VII, VIII, IX y X, tuvieron riesgos mayores al promedio, con una tasa media de mortalidad de 13,9 por $100.000 \mathrm{~h}$ (IC 95\%: 12,914,8), siendo las más altas las regiones IX y X, las que, además, presentan altos índices de ruralidad, pobreza y mayor proporción de población mapuche $^{19}$ (Figura 3).

Tabla 1. N úmero de casos y tasa de mortalidad estandarizada (x $100.000 \mathrm{~h}$ ) por cáncer de vesícula biliar, C hile, período 1985-2002

\begin{tabular}{|lcrrrrr|}
\hline & \multicolumn{2}{c}{ Total } & \multicolumn{2}{c}{ Hombres } & \multicolumn{2}{c|}{ Mujeres } \\
Año & Muertes & Tasa & Muertes & Tasa & Muertes & Tasa \\
\hline 1985 & 1.140 & 11,0 & 283 & 6,7 & 857 & 15,3 \\
1986 & 1.178 & 11,2 & 332 & 7,6 & 846 & 14,9 \\
1987 & 1.141 & 10,4 & 280 & 6,2 & 861 & 14,6 \\
1988 & 1.265 & 11,3 & 336 & 7,2 & 929 & 15,4 \\
1989 & 1.305 & 11,3 & 311 & 6,6 & 994 & 16,1 \\
1990 & 1.342 & 11,4 & 342 & 6,9 & 1.000 & 15,9 \\
1991 & 1.453 & 11,9 & 357 & 7,0 & 1.096 & 16,8 \\
1992 & 1.414 & 11,2 & 373 & 7,1 & 1.041 & 15,4 \\
1993 & 1.457 & 11,3 & 398 & 7,4 & 1.059 & 15,2 \\
1994 & 1.552 & 11,6 & 428 & 7,6 & 1.124 & 15,6 \\
1995 & 1.628 & 11,9 & 434 & 7,6 & 1.194 & 16,2 \\
1996 & 1.667 & 11,6 & 431 & 7,2 & 1.236 & 16,0 \\
1997 & 1.711 & 11,6 & 440 & 7,1 & 1.271 & 16,1 \\
1998 & 1.707 & 11,2 & 424 & 6,7 & 1.283 & 15,8 \\
1999 & 1.770 & 11,5 & 461 & 7,1 & 1.309 & 15,8 \\
2000 & 1.842 & 11,5 & 495 & 7,3 & 1.347 & 15,8 \\
2001 & 1.733 & 10,5 & 462 & 6,6 & 1.271 & 14,5 \\
2002 & 1.878 & 11,0 & 477 & 6,7 & 1.401 & 15,3 \\
Total & 27.183 & 11,3 & 7.064 & 7,0 & 20.119 & 15,6 \\
\hline
\end{tabular}




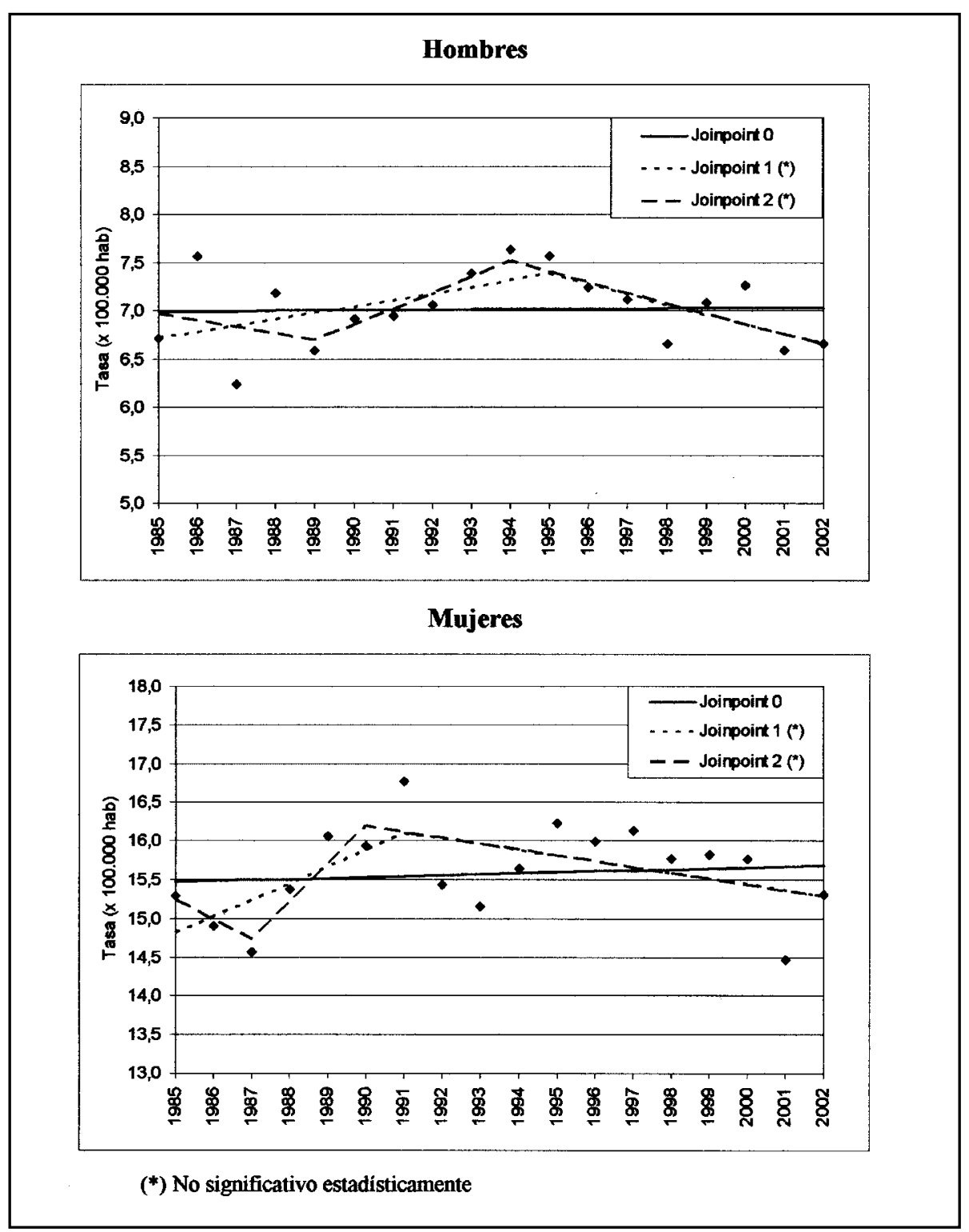

Figura 1. Mortalidad por cáncer de vesícula biliar, Chile 1985-2002. Análisis de punto de cambio de la tasa de mortalidad estandarizada según sexo.

Aunque en todas las regiones las mujeres presentaron un exceso de riesgo de cáncer vesicular con respecto a los hombres, el exceso fue menor en las regiones I, XI y XII, donde el RR de mujeres versus hombres, ajustado por edad, fue de 1,6 (95\% IC: 1,3-1,9). Estas tres regiones son las más extremas del país y con menor proporción de población mapuche.

Entre los años 2003 y 2004 se realizaron en Chile un promedio de 22.701 colecistectomías anuales, equivalentes a una tasa de 150,1 colecistectomías por 100.000 h. En el año 2004 quedaron 
Tabla 2. Cáncer de vesícula biliar, tasa de mortalidad específica por grupo de edad y sexo. Chile, promedio período 1985-2002

\begin{tabular}{|lccc|}
\hline $\begin{array}{l}\text { Edad } \\
\text { (años) }\end{array}$ & Hombres & $\begin{array}{c}\text { Tasa de mortalidad x } 100.000 \mathrm{~h} \\
\text { Mujeres }\end{array}$ & Total \\
\hline $0-4$ & 0,00 & 0,00 & 0,00 \\
$5-9$ & 0,00 & 0,00 & 0,00 \\
$10-14$ & 0,02 & 0,00 & 0,01 \\
$15-19$ & 0,00 & 0,01 & 0,00 \\
$20-24$ & 0,01 & 0,04 & 0,02 \\
$25-29$ & 0,10 & 0,17 & 0,14 \\
$30-34$ & 0,24 & 1,04 & 0,64 \\
$35-39$ & 0,79 & 3,41 & 2,10 \\
$40-44$ & 2,09 & 8,01 & 5,08 \\
$15-49$ & 4,06 & 17,54 & 10,93 \\
$50-54$ & 7,56 & 29,03 & 18,62 \\
$55-59$ & 15,26 & 44,04 & 30,41 \\
$60-64$ & 25,04 & 62,32 & 45,11 \\
$65-69$ & 39,88 & 83,44 & 64,02 \\
$70-74$ & 61,81 & 115,51 & 92,71 \\
$75 \mathrm{y}+$ & 107,83 & 163,10 & 142,28 \\
\hline
\end{tabular}

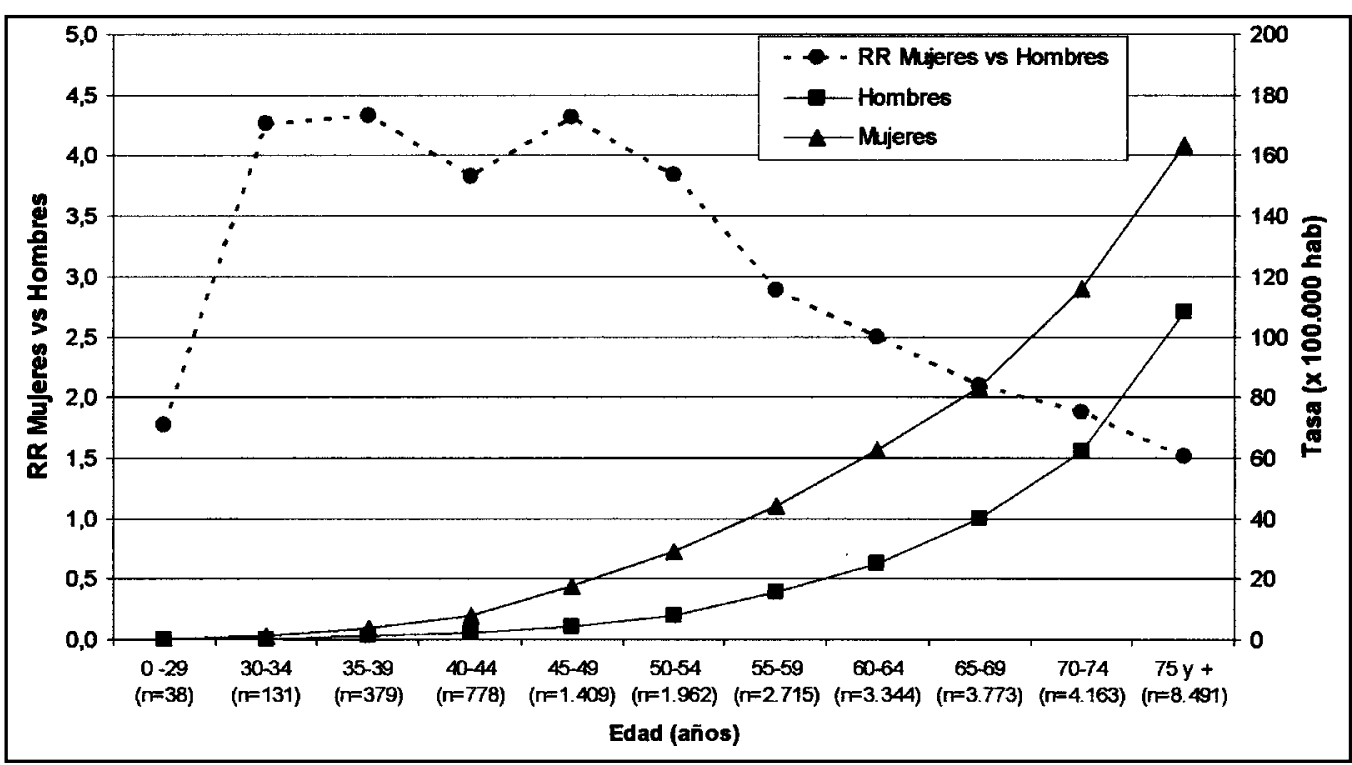

Figura 2. Tasa de mortalidad específica cáncer vesicular y riesgo relativo mujeres versus hombres, Chile 1985-2002.

sin operar, y en espera de cirugía, un total de 10.213 pacientes a nivel nacional*, lo que representa una tasa de 6,8 pacientes en espera por 10.000 h (Tabla 3). Se encontró una relación directa, sin alcanzar significación estadística, entre la mortalidad regional y la tasa de pacientes en lista de espera $\left(\mathrm{R}^{2}: 0,27\right)$ (Figura 4$)$.

\footnotetext{
* Estos datos consideran 26 de los 28 Servicios de Salud del país más el Hospital Padre Hurtado. No se han considerado los Servicios de Salud de Araucanía Norte y Osorno por poseer información incompleta.
} 


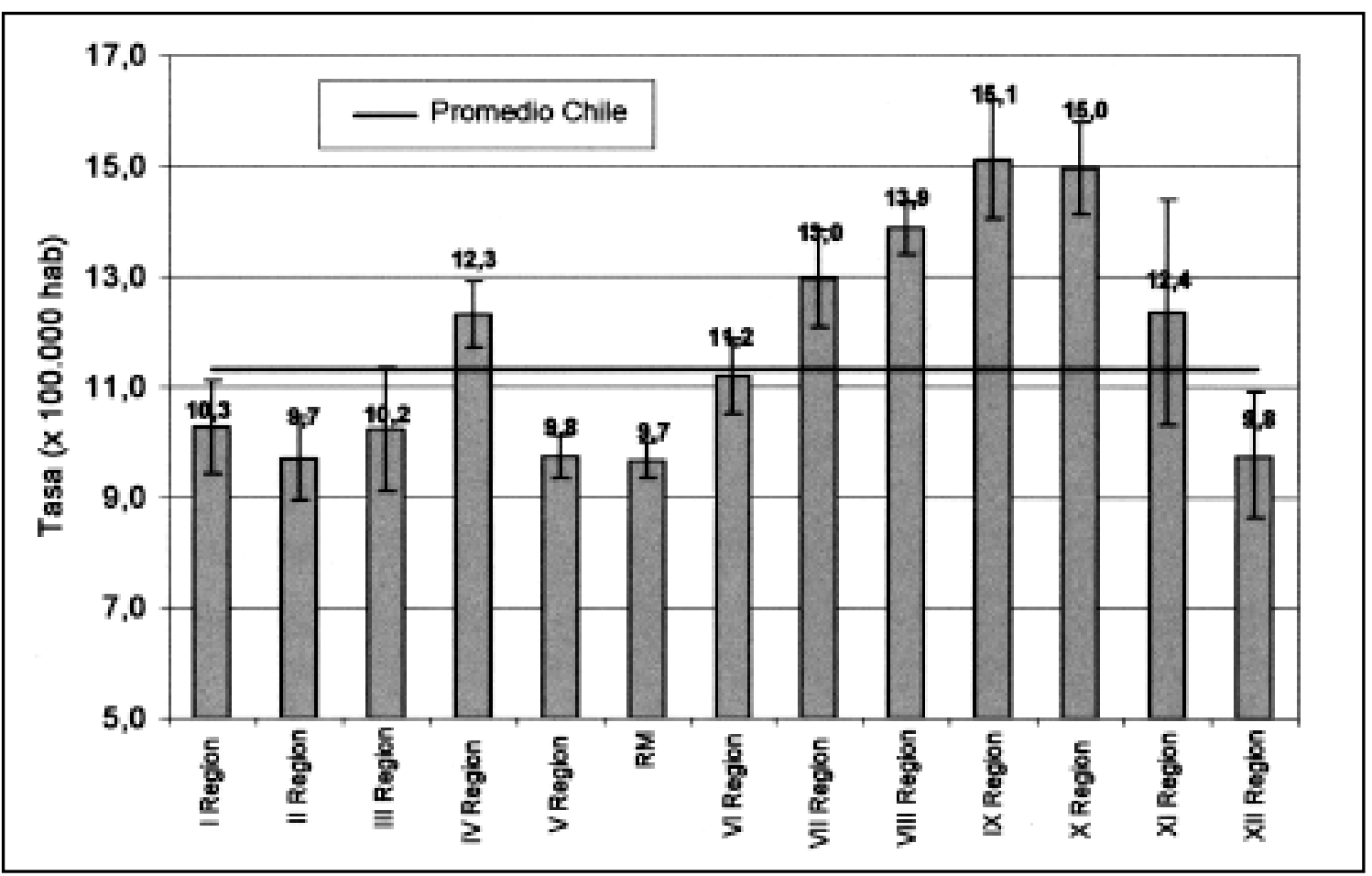

Figura 3. Mortalidad por cáncer de vesícula biliar, tasa media estandarizada (IC 95\%). Regiones de Chile 1985-2002.

Tabla 3. N úmero y tasa de colecistectomías por regiones promedio 2003-2004 y número y tasa de pacientes en espera de colecistectomía año 2004. 13 regiones de C hile

\begin{tabular}{|lrrrr|}
\hline Región & $\begin{array}{r}\text { № de colecistectomías } \\
\text { (Tasa x } 100.000 \mathrm{~h})\end{array}$ & $\begin{array}{r}\text { Pacientes en Espera de cirugía } \\
\text { (Tasa x } 10.000 \mathrm{~h})\end{array}$ \\
\hline I & 502 & $(122,0)$ & 208 & $(5,1)$ \\
II & 572 & $(119,1)$ & 482 & $(10,0)$ \\
III & 324 & $(114,5)$ & 3 & $(0,1)$ \\
IV & 1.144 & $(192,6)$ & 28 & $(0,5)$ \\
V & 2.583 & $(161,8)$ & 869 & $(5,4)$ \\
RM (a) & 7.714 & $(122,9)$ & 2.642 & $(4,2)$ \\
VI & 1.098 & $(135,7)$ & 257 & $(3,2)$ \\
VII & 1.342 & $(144,2)$ & 762 & $(8,2)$ \\
VIII & 3.485 & $(173,8)$ & 3.256 & $(16,2)$ \\
IX (b) & 1.420 & $(227,8)$ & 560 & $(9,0)$ \\
X (c) & 1.859 & $(217,0)$ & 940 & $(11,0)$ \\
XI & 235 & $(240,4)$ & 186 & $(19,0)$ \\
XII & 425 & $(265,6)$ & 20 & $(1,2)$ \\
Chile & 22.701 & $(150,1)$ & 10.213 & $(6,8)$ \\
\hline
\end{tabular}

Fuente: elaboración propia a partir de listas de espera de la Secretaría Plan Auge. MINSAL.

(a): Incluye Hospital Padre Hurtado. $\left({ }^{b}\right)$ No incluye S.S. Araucanía Norte. $\left(^{c}\right)$ No incluye S.S. Osorno. 


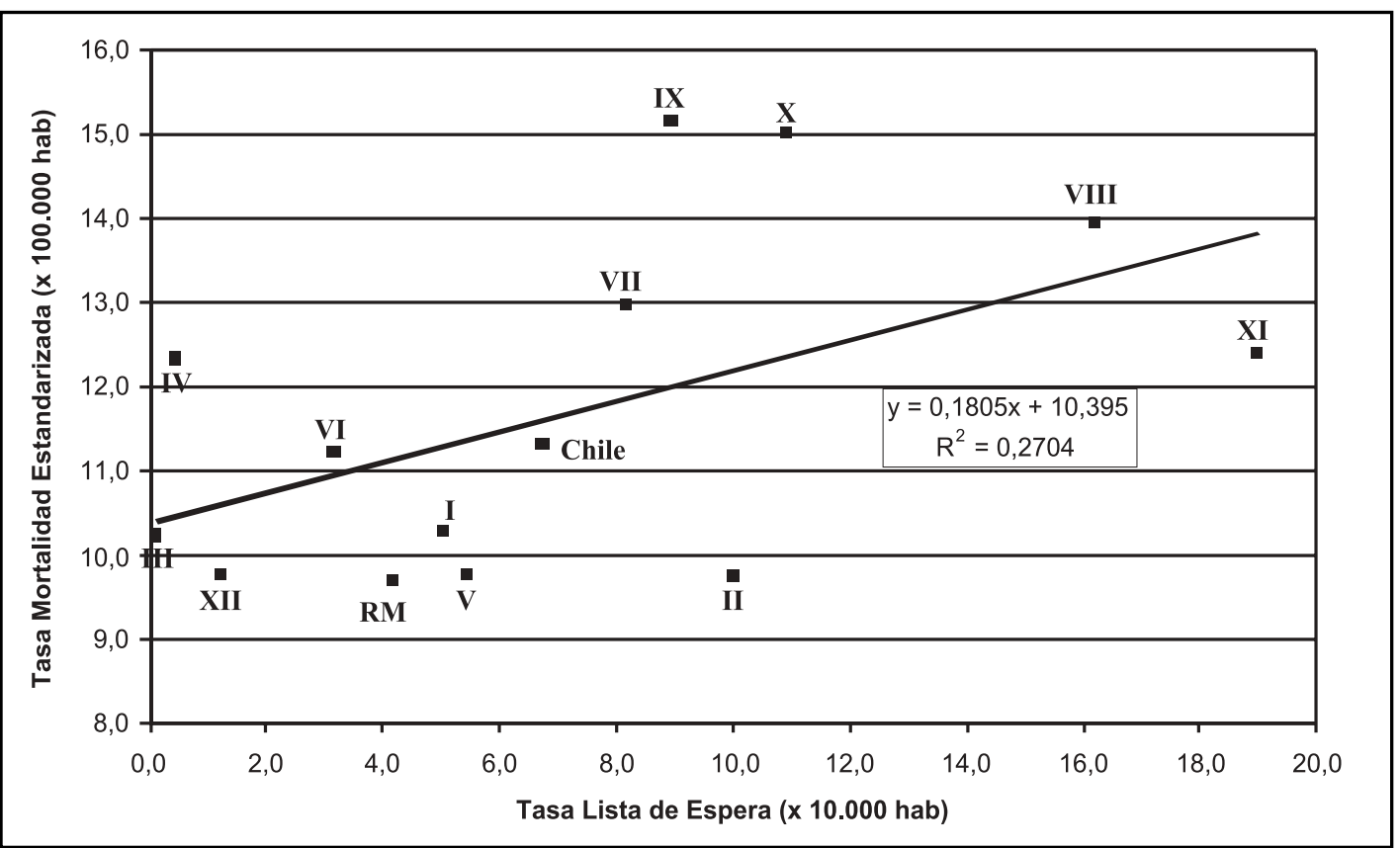

Figura 4. Tasa de mortalidad estandarizada por cáncer vesicular promedio 1985-2002 versus tasa de personas en espera de colecistectomía año 2004. 13 regiones de Chile.

Se exploró la relación del Producto Interno Bruto (PIB) per cápita con la mortalidad por cáncer vesicular en 23 países de América y Europa ${ }^{4,6,7}$, encontrándose una relación inversa (a mayor PIB menor mortalidad), con un coeficiente de determinación $\left(\mathrm{R}^{2}\right)$ de 0,42 a 0,48 en hombres y mujeres, respectivamente (Figura 5). Chile se sale de la línea de tendencia tanto en hombres como en mujeres, presentando tasas de mortalidad 2,8 veces más altas que las esperadas para su PIB en mujeres y 2,4 veces en hombres.

\section{DisCUSIÓN}

A nivel nacional, la tasa de mortalidad estandarizada por cáncer vesicular ha permanecido prácticamente constante en los 18 años analizados, tanto para hombres como para mujeres. La leve disminución al final del período hace necesario seguir la evolución de la tasa para determinar un cambio en la tendencia. La persistencia de alta mortalidad, a pesar de mejores condiciones económicas y sanitarias, es discordante con la expe- riencia en otros países ${ }^{7}$ y sugiere la presencia de otros factores de riesgo en Chile.

El exceso de riesgo de cáncer vesicular de mujeres vs hombres en Chile es superior al descrito en Europa $^{7}$ y comparable al de los hispánicos y nativos de Norteamérica y a otros países sudamericanos ${ }^{4,6}$. Esta mayor susceptibilidad para este cáncer de las mujeres nativas del norte y sur de América, quienes también presentan un exceso de riesgo de colelitiasis, se explicaría por ancestros comunes ${ }^{20}$, sugiriendo un componente genético en el exceso de riesgo de las mujeres chilenas. El máximo diferencial de riesgo ocurre en la edad fértil de la mujer y disminuye en el periodo posmenopáusico, sugiriendo un efecto importante de los estrógenos. Esto ha sido descrito por otros autores, que también encontraron un mayor riesgo de cáncer de vesícula asociado con menarquia precoz, menopausia tardía, múltiples embarazos, la terapia de reemplazo hormonal que se ha asociado a un aumento de la litiasis vesicular y un mayor riesgo de cáncer de vesícula $8,9,21$.

El factor causal más importante del cáncer de vesícula biliar es la colelitiasis ${ }^{10,22}$, se estima que 


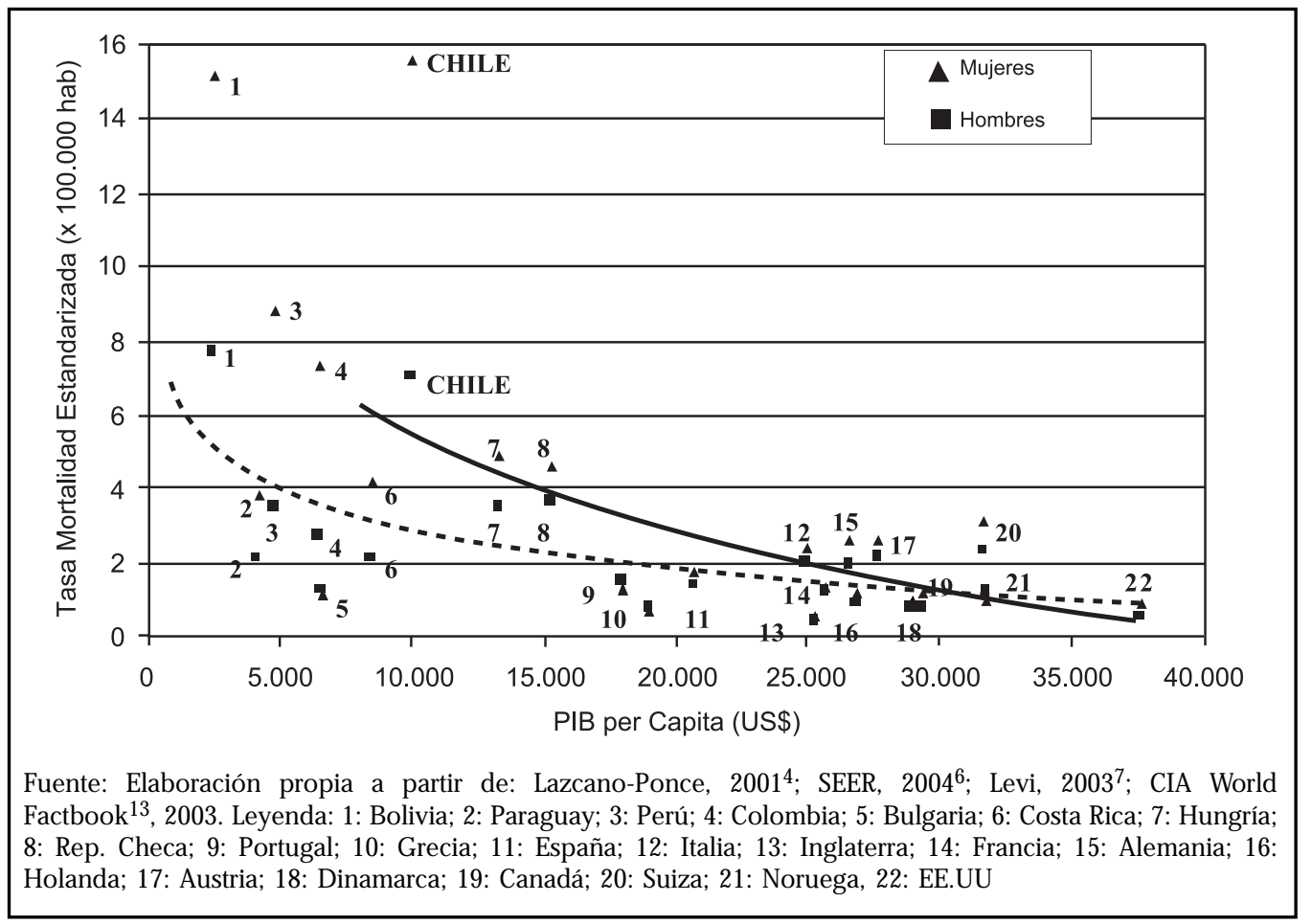

Figura 5. Tasa de mortalidad estandarizada por cáncer vesicular vs PIB per cápita, año 2000 en 23 países de Europa y América.

en Chile esta patología está presente en $47 \%$ de las mujeres adultas 23 , diversos estudios muestran que del total de las vesículas operadas por colelitiaisis, entre 3 y $4 \%$ presentan cáncer vesicular en distintos estadios en el examen anatomopatológico ${ }^{24}$. Por ello, se ha planteado que la colecistectomía es la intervención fundamental para disminuir la mortalidad por cáncer vesicular en Chile $7,25,26$.

Hasta recientemente no había datos sobre el número de colecistectomías en el país. Chianale et $a^{26}$ estimaron la tasa de colecistectomías a partir del número de egresos hospitalarios relacionados a enfermedad litiásica de la vía biliar, y observaron que entre 1980 y 1988 la tasa disminuyó, presentando un valor promedio de 290 cirugías biliares por $100.000 \mathrm{~h}$ en el periodo. Ellos encontraron una correlación significativa entre la disminución de las cirugías y el aumento en la mortalidad por cáncer vesicular. Estimó que era necesario aumentar las colecistectomías en 100 cirugías por
$100.000 \mathrm{~h}$ para disminuir la mortalidad en 1 muerte por $100.000 \mathrm{~h}$. Siguiendo a Chianale et al, estimamos para 2001 una tasa de colecistectomías de 318 por $100.000 \mathrm{~h}^{27}$, sólo $10 \%$ más alta que lo observado por ellos para la década 1980-89. Este discreto aumento de las cirugías sería insuficiente para impactar en la mortalidad y explicaría su estancamiento.

Pese al aumento del número de colecistectomías a nivel nacional, aún persiste un déficit en las regiones de mayor riesgo de cáncer vesicular, que no logran disminuir la brecha entre la oferta y la necesidad de cirugía que se arrastra históricamente. Aunque la relación entre la mortalidad regional y la tasa de pacientes en espera de cirugía vesicular, no alcanzó significación estadística, sugiere que en las regiones con mayor mortalidad por cáncer vesicular la oferta actual de colecistectomías es aún insuficiente. Esto ya lo habían observado Pérez-Ayuso et $\mathrm{al}^{28}$ al reportar que la población mapuche, la más afectada por el 
cáncer vesicular, presenta una alta prevalencia de colelitiasis sintomáticas sin resolución quirúrgica.

El costo de una colecistectomía simple es de $\$ 300.000^{25}$, la inversión total para resolver los casos en espera es de US\$ 5,3 millones, por sobre el costo anual del programa de colecistectomías. $\mathrm{Si}$ se dispusiera de estos fondos, se aumentaría las colecistectomías en una tasa de 65 cirugías por $100.000 \mathrm{~h}$, lo que según Chianale et $\mathrm{al}^{26}$ podría disminuir la tasa de mortalidad en 0,7 muertes por 100.000 h, es decir se evitarían 105 muertes por año.

Las tres regiones con mayores tasas de mortalidad estandarizada (regiones VIII, IX y X) son zonas principalmente agrícolas, con altos índices de ruralidad, con los menores ingresos per cápita del país y en las cuales hay una mayor proporción de población mapuche, lo que apoya la hipótesis planteada por Lazano-Ponce et $\mathrm{al}^{4}$ y Galman et $\mathrm{al}^{29}$ con respecto a la mayor tendencia a la colelitiasis y al cáncer de vesícula de la población originaria americana. Pero también son las áreas más pobres y con menor acceso a la cirugía biliar. Estas regiones concentrarían una mayor suscepti-

\section{REFERENCIAS}

1. GLOBOCAN. Cancer Incidence, Morality and Prevalence Worldwide. IARC Cancer Base №5, 2000.

2. WHO, World Cancer Report, World Health Organization - International Agency for Research on Cancer, IARC Press, 2003.

3. O’Brien K, Cokrinides V, Jemal A, Cardinez A, Murray T, SAmuels A et al. Cancer Statistics for Hispanics, 2003. CA Cancer J Clin 2003; 53: 20826.

4. Lazcano-Ponce E, Miquel J, Muñoz N, HerRero R, FerRecCio C, Wistuba I et al. Epidemiology and Molecular Pathology of Gallbladder Cancer. CA Cancer J Clin 2001; 51: 349-64.

5. MINSAL Registro Poblacional del Cáncer. Provincia de Valdivia, 1995-2002. Ministerio de Salud, Chile, 2004.

6. SEER. Surveillance, Epidemiology and End Results Program. 2004, wwww.seer.cancer.gov bilidad genética y vulnerabilidad social. Lo contrario ocurre en las regiones con menores tasas de mortalidad (regiones XII, II y RM), donde los mayores ingresos per cápita se acompañan de mejor acceso a salud y menor proporción de población mapuche.

Nuestro estudio demuestra que el cáncer vesicular sigue siendo un importante problema médico y de salud pública en Chile y que ya existe información suficiente para intervenir y disminuir, de modo importante, el daño individual y social producto de esta enfermedad. El desafío del sector salud es ampliar de manera importante y urgente la oferta de colecistectomías, para la población sintomática biliar de las regiones de alto riesgo. Una vez resueltos estos casos sintomáticos, se puede afinar criterios de tamizaje para identificar grupos de mayor riesgo que se beneficiarían con esta operación.

Aún hay preguntas importantes sobre la naturaleza del cáncer vesicular y Chile presenta condiciones privilegiadas para aportar a su conocimiento por la magnitud y heterogeneidad de la ocurrencia en el país.

7. Levi F, Lucchini F, Negri E, La Vecchia C. The recent decline in gallbladder cancer mortality in Europe. Eur J Cancer Prev 2003; 12: 265-7.

8. Pandey M, Shukia VK. Lifestyle, parity, menstrual and reproductive factors and risk of gallbladder cancer. Eur J Cancer Prev 2003; 12: 269-72.

9. Mitchell JL, Walsh J, Wang-Cheng R, Hardman JL. Postmenopausal hormone therapy: a concise guide to therapeutic uses, formulations, risks and alternatives. Prim Care 2003; 30: 671-96.

10. PANDEY M. Risk factors for gallbladder cancer: a reappraisal. Eur J Cancer Prev 2003; 12: 15-24.

11. Ferreccio C, Chianale J, González C, Nervi F. Epidemiología descriptiva del cáncer digestivo en Chile (1982-1991): Una aproximación desde la mortalidad, Edit Alfa Beta, Santiago, 1995: 119131.

12. MINSAL, Ministenio de Salud de Chile (www.minsal.cl)

13. INE, Instituto Nacional de Estadísticas, 2005 (www.ine.cl) 
14. World Factbook, 2005 (www.odci.gov/cia/publications/factbook/index.html)

15. Doш R, Cook P. Summarizing indices for comparison of cancer incidence data. Int J Cancer 1967; 2: 269-79.

16. Kim HJ, Fay MP, Feuer EJ, Midthune DN. Permutation test for joinpoint regression with applications to cancer rates. Stat Med 2000; 19: 335-51.

17. National Cancer Institute. Joinpoint Regression Program, Version 2.7. September 2003.

18. SPSS for Windows version 13.0. SPSS Inc.

19. MIDEPLAN, Ministerio de Planificación y Cooperación, 2005 (www.mideplan.cl)

20. CAREY MC, Paigen B. Epidemiology of the American Indians' burden and its likely genetic origins. Hepatology 2002; 36: 781-91.

21. Gaums S, Negri E, Chatenoud L, Bosetti C, Franceschi S, LA VeCCHIA C. Post menopausal hormonal therapy and gallbladder cancer. IntJ Cancer 2002; 99: 762-3.

22. Misra S, Chaturvedi A, Sharma I. Cancinoma of the gallbladder. Lancet Oncol 2003; 4: 167-76.

23. Fernández M, Csendes A, Yarmuch J, Díaz H, Silva J. Management of common bile duct stones: the state of the art in 2000. Int Surg 2003; 88: 159-63.
24. Roa I, De Aretxabala X, Araya J. Carcinoma incipiente de la vesícula biliar: Estudio clínicopatológico y pronóstico de 196 casos. Rev Méd Chile 2001; 129: 1113-20.

25. SERRA I. [Has gallbladder cancer mortality decreased in Chile?]. Rev Méd Chile 2001; 129: 1079-84.

26. Chianale J, Valdivia G, Del Pino G, Nervi F. Mortalidad por cáncer vesicular en Chile y su relación con las tasas de colecistectomía. Análisis de la última década. Rev Méd Chile 1990; 118: 1284-8.

27. MINSAL, Ministerio de Salud de Chile, Estadísticas de Egresos Hospitalarios, año 2001. (www.minsal.cl)

28. Pérez-Ayuso RM, Hernández V, González B, CarvaCHO C, NavarRete C, Alvarez M et al. [Natural history of cholelithiasis and incidente of cholecystectomy in an urban and a Mapuche rural area]. Rev Méd Chile 2002; 130: 723-30.

29. Galman C, Miquel JF, Pérez RM, Einarsson C, Stahie L, Marshall G et al. Bile acid synthesis is increased in Chilean Hispanics with gallstones and in gallstone high-risk Mapuche Indians. Gastroenterology 2004; 126: 741-8. 